\title{
A Research of Grid Manufacturing and Its Application in Custom Artificial Joint
}

\author{
Li Chen ${ }^{1}$, Hong Deng ${ }^{1}$, Qianni Deng ${ }^{2}$, and Zhenyu Wu ${ }^{1}$ \\ ${ }^{1}$ Shanghai Jiao Tong University, School of Mechanical and Power Engineering, \\ Shanghai 200030 , \\ 62932905 Shanghai, China \\ \{chen_li, denghong76, wzy\} @sjtu.edu.cn \\ ${ }^{2}$ Shanghai Jiao Tong University, Department of Computer and Science, Shanghai 2000 30, \\ 62932632 Shanghai, China \\ deng-qnacs.sjtu.edu.cn
}

\begin{abstract}
This paper presents the framework of Grid Manufacturing, which neatly combines Grid technology with the infrastructure of advanced manufacturing technology. It studies the Grid-oriented knowledge description and acquisition, and constructs the distributed Knowledge Grid model. It also deals with the protocol of node description in collaborative design, and builds up the distributed collaborative design model. And the research on the protocol and technology of node constructing leads to the collaborative production model of Grid Manufacturing. Finally, the framework of Grid Manufacturing is applied in the design and manufacturing of custom artificial joint and the joint product is produced more efficiently.
\end{abstract}

\section{Introduction}

With the rapid technological innovations of the networked manufacturing, much more is learned about the inherent limitations of the network technology. Grid is regarded as the next generation Internet as well as Grid Manufacturing is then presented as an advanced solution for the bottleneck of networked manufacturing. The research on Grid will build up solid theoretical and technological fundaments to realize a great stride in manufacturing [1-4].

\section{Data and Knowledge Management of Grid Manufacturing}

Data and knowledge management of Grid Manufacturing includes the following steps:

Firstly analyzing the storage mode and structure of heterogeneous data on the Grid nodes; then building up the general and open knowledge description, internal encapsulation protocol and exchange standard, and various information and knowledge 
required in Grid Manufacturing encapsulation (including the heterogeneous database, design and operating know-how, thinking process on various nodes, etc.); finally displaying these knowledge by the uniform external interactive protocols and interfaces[5].

\section{Collaborative Design and Production Model of Grid Manufacturing}

The distributed design mechanism based on Grid Manufacturing technology is seeking to enhance the interactions of collaborative design between the dynamic union of enterprises to the level of high efficiency, high speed, large scale, and massive data traffic [6].

The construction protocol and technology of manufacturing nodes is to realize the high performance scheduling and dynamic collaboration of Grid Manufacturing resources.

The kernel of the collaborative production model, the resources scheduling and manufacturing collaboration will be realized by the five-level Grid structure and related functional modules. With the functions and services provided by the five-level Grid structure, the manufacturing collaboration can be finally achieved when these sub-models are realized by the technical support of the corresponding level. The production collaboration model is shown in Fig. 1.

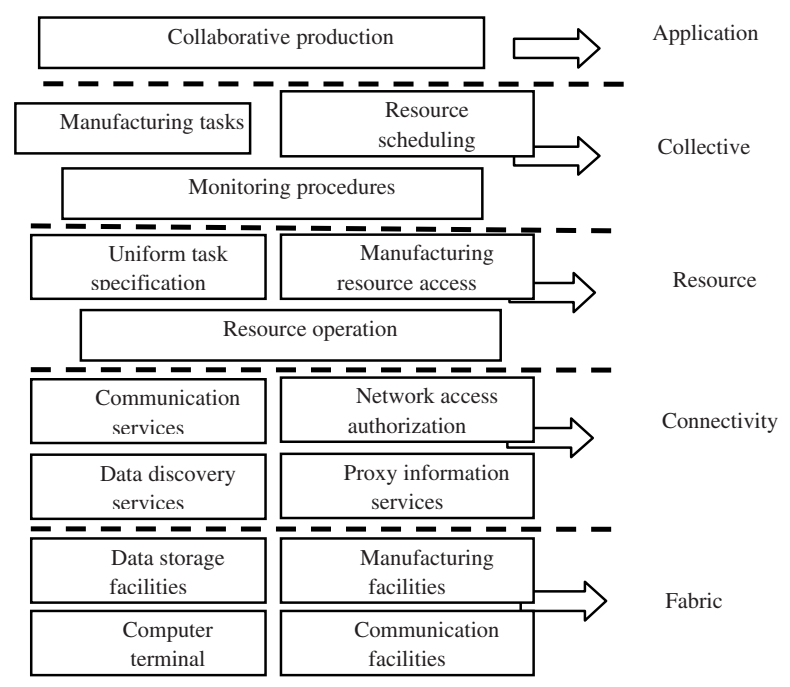

Fig. 1. Collaborative production model 

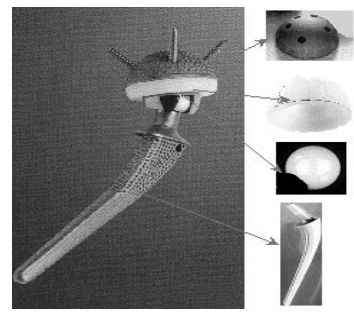

cup

liner

ball

handle

Fig. 2. A product of custom artificial hip joint

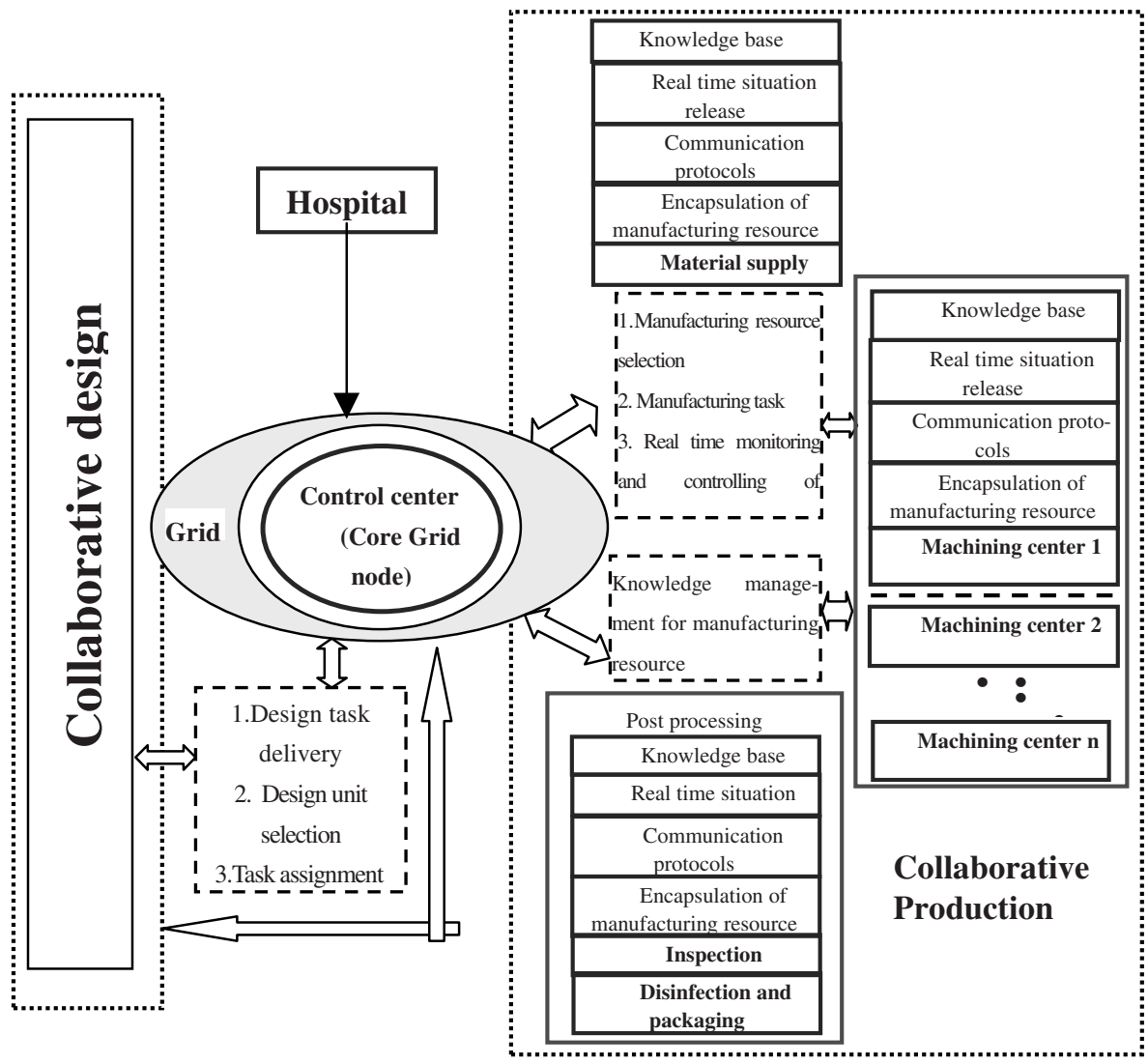

Fig. 3. The workflow of custom artificial joint in Grid Manufacturing environment 


\section{An Application of Grid Manufacturing in the Custom Artificial Joint}

Custom artificial joint is a special product designed for perfectly matching an individual's medullary cavity, whose demands are unforeseeable and urgent[7]. Once a custom artificial joint prosthesis is demanded, it should be supplied as quickly as possible. Everyone is absolutely individualized.A product of custom artificial hip joint is illustrated in Fig.2.

The workflow of custom artificial joint in Grid Manufacturing environment is illustrated in Fig.3.

\section{Conclusion}

This research presents the concept of Grid Manufacturing in the world, which is the next-generation technology subsequent to the networked manufacturing. Along with more work done in the Grid Manufacturing environment, the defects with networked manufacturing will be conquered. The Grid Manufacturing framework will push forward greatly the development of advanced manufacturing technology. Based on Grid Manufacturing environment, the design and manufacturing of custom artificial joint product has been improved obviously. One hand, collaborative design of joint product among doctors, patient and engineers has been more efficient. On the other hand, the flexibility of production of joint product also has been improved and the cost of custom artificial joint has been reduced.

\section{References}

1. Xingjun Chu, Yuqing Fan: The research of PDM based on Web. Journal of Beijing Aeronautics and Astronauts University 2 (1999) 205-207

2. Xucan Chen, Yuxing Peng, Sikun Li: PDM of CAD Collaborative Design based on C-S. Computer Engineering and Design (1998) 53

3. Dan Wu, Xiankui Wang, Zhiqiang Wei et al.: The Distributed PDM based on Collaborative Service Environment. Journal of Tsinghua University (Science and Technology) 6 (2002) 791-781

4. Zhiqiang Wei, Xiankui Wang, Chengyin Liu et al.: The Distributed PDM under the Environment of Agile Manufacturing. Journal of Tsinghua University(Science and Technology) 8 (2000) 45

5. H.Zhuge: A Knowledge Grid Model and Platform for Global Knowledge Sharing. Expert System with Applications, Vol. 22. no.4 (2002)

6. G. von Laszewski, I. Foster, J. Gawor et al.: Designing Grid-based problem solving environments and portals. Proceedings of the 34th Annual Hawaii International Conference on System Sciences, IEEE Press (2001)

7. Shang Peng: The multi-agent design system of custom artificial hip joint. Journal of

8. Chinese Biomedical Engineering 2 (2001) 